\title{
Correlation of Immunoglobulins and Lymphocytes Levels With the Clinical and Microbiological Response of Septic Patients With Gram-Negative Bacteremia
}

\author{
Diamanto Aretha ${ }^{\mathrm{a}, \mathrm{d}}$, Katerina Leukaditou ${ }^{\mathrm{a}}$, Fotini Fligou ${ }^{\mathrm{a}}$, Karolina Akinosoglou ${ }^{\mathrm{b}}$, \\ Alexandros Spyridonidis ${ }^{\mathrm{c}}$, Alexandra Nikolopoulou ${ }^{\mathrm{a}}$, Stelios F. Assimakopoulos ${ }^{\mathrm{b}}$
}

\begin{abstract}
Background: Immunoglobulins (Igs) and cells of the innate and adaptive immune systems play a critical role in a host's response to sepsis. The aim of this study was to investigate the possible differences in the levels of Igs, white blood cells (WBCs), and T and B lymphocytes cells in relation to the microbiological and clinical responses of patients with sepsis or septic shock from carbapenem nonsusceptible Gram-negative bacteria (CnS-GNB).
\end{abstract}

Methods: This pilot cohort study involved 24 hospitalized patients with sepsis or septic shock due to bacteremia from CnS-GNB. The microbiological and clinical responses of the patients were evaluated in relation to their blood levels of $\operatorname{IgA}, \operatorname{IgE}, \operatorname{IgM}$ and $\operatorname{IgG}$, as well as WBCs and subpopulations of $\mathrm{T}$ and $\mathrm{B}$ cells upon sepsis diagnosis. A microbiological response was determined as clearance of bacteremia at 14 days of active antibiotic treatment for the isolated bacterial pathogen. Clinical response was defined as the resolution of all clinical and laboratory signs of infection and sepsis at 14 days of active antibiotic treatment for the isolated pathogen.

Results: From the 24 patients included in the study 18 (75\%) and six patients $(25 \%)$ presented and did not present microbiological response respectively, while 16 patients presented clinical response $(64 \%)$ and eight patients (36\%) did not have clinical response. The levels of the Igs did not show statistically significant differences between patients with sepsis from CnS-GNB bacteremia who exhibited microbiological or clinical response. There were also no statistically significant differences in the levels of WBCs and the subpopulations of $\mathrm{T}$ and $\mathrm{B}$ cells levels for these patients $(\mathrm{P}>0.05)$. According to

Manuscript submitted December 8, 2020, accepted December 30, 2020

Published online January 12, 2021

antensive Care Medicine, University General Hospital of Patras, 26504 Patras, Greece

bInternal Medicine and Infectious Diseases, University General Hospital of Patras, 26504 Patras, Greece

'Hematology Division, BMT Unit, University General Hospital of Patras 26504 Patras, Greece

${ }^{\mathrm{d} C o r r e s p o n d i n g ~ A u t h o r: ~ D i a m a n t o ~ A r e t h a, ~ D e p a r t m e n t ~ o f ~ A n e s t h e s i o l o g y ~ a n d ~}$ Intensive Care Medicine, University General Hospital of Patras, 26504 Rion, Patras, Greece. Email: adaretha@yahoo.gr

doi: https://doi.org/10.14740/jocmr4409 this pilot study, peripheral blood Igs and lymphocyte subpopulations levels do not affect the clinical and microbiological response of septic patients with bacteremia from CnS-GNB.

Conclusions: In patients with sepsis or septic shock from CnSGNB, there were no differences in the levels of Igs, circulating WBCs and $\mathrm{T}$ and $\mathrm{B}$ cells subpopulations between those with microbiological or clinical response and non-responders.

Keywords: Sepsis; Immunoglobulins; Lymphocytes; Carbapenem non-susceptible; Gram-negative bacteria

\section{Introduction}

Antimicrobial resistance in hospital settings is a worldwide threat and a global public health problem [1-3]. In Greece, nosocomial infections from carbapenem non-susceptible Gramnegative bacteria (CnS-GNB) are serious and complex issues, especially in patients in intensive care unit (ICU). Nosocomial infections cause a significant increase in morbidity and mortality rates, as well as in the duration and costs of hospitalization stays of patients.

Sepsis is an acute, life-threatening, exaggerated systemic host response to infection that is associated with organ dysfunction, hyperinflammation and immunosuppression [4-6]. To determine the effects of sepsis on the immune system, in vitro and in vivo models have been used to study the levels of Igs, white blood cells (WBCs), and T and B lymphocytes [7-11]. Most patients with sepsis present with low concentrations of Igs. Immunomodulatory therapies with intravenous Ig (IVIG) or IgMenriched IVIG (IVIGAM) are considered as potential adjuvant therapy for these patients [12-15]. Sepsis is characterised by functional, phenotypic and quantitative changes in $\mathrm{T}$ and $\mathrm{B}$ lymphocytes. Sepsis-induced apoptosis leads to lymphopenia of most types of T cells, including CD4, CD8 and natural killer (NK) cells, except T regulatory cells, which favor immunosuppression [16-18]. Furthermore, circulating levels of B lymphocytes correlate with the incidence of nosocomial infection [7].

To our knowledge, the correlation between the levels of Igs, WBCs and lymphocytes' subpopulations with the microbiological and/or clinical response of patients with sepsis from $\mathrm{CnS}-\mathrm{GNB}$ has never been evaluated. The aim of the present 
study was to examine the impact of the levels of $\operatorname{IgM}, \operatorname{IgG}, \operatorname{Ig} \mathrm{A}$ and IgE and the circulating subpopulations of WBCs and T and $\mathrm{B}$ cells on the microbiological and clinical response of septic ICU patients with bacteremia from $\mathrm{CnS}-\mathrm{GNB}$.

\section{Materials and Methods}

\section{Design}

This single-centre, prospective, observational, cohort pilot study was performed between June 2018 and April 2019 in the general ICU of University General Hospital of Patras (UGHP), Greece. The UGHP Scientific and Ethics Committee approved the study (number: 255/15.05.18). The data were collected after consent was obtained from the closest relative, as the patients were unable to provide consent due to the severity of their disease. This study was conducted in compliance with all the applicable institutional ethical guidelines for the care and welfare.

Inclusion criteria were patients $>18$ years of age, presenting sepsis and/or septic shock caused by bacteremia from $\mathrm{CnS}$ GNB Acinetobacter baumannii, Klebsiella pneumonia and Pseudomonas aeruginosa. The definitions of sepsis and septic shock were based on the Sepsis-3 criteria [5]. Upon sepsis or septic shock diagnosis blood was drawn for culture and study's analyses, and empiric antibiotic treatment was started within $1 \mathrm{~h}$. When a CnS-GNB (Acinetobacter baumannii, Klebsiella pneumonia and Pseudomonas aeruginosa) was isolated in blood culture the patient was enrolled in the study for analyses of Igs levels and circulating WBCs and lymphocyte subpopulations at the onset of bacteremia. Appropriateness of antibiotic treatment was reassessed by an infectious diseases specialist upon the availability of the susceptibility tests results and within the first 7 days after blood culture collection (targeted therapy). Patients enrolled should have been treated with at least one active in vitro agent for Acinetobacter baumannii or Pseudomonas aeruginosa or at least two active in vitro agents for Klebsiella pneumonia, according to the susceptibility test results. Monotherapy with tigecycline (even at high dose of $200 \mathrm{mg}$ loading dose, followed after $12 \mathrm{~h}$ by $100 \mathrm{mg}$ bid) was considered inactive for Acinetobacter baumannii bacteremia. The exclusion criteria were isolation of more than one pathogens, human immunodeficiency virus (HIV) infection, primary immunodeficiency or neutropenia (i.e., $<1,000$ neutrophils/ $\mathrm{mm}^{3}$ ), or chronic corticosteroid therapy (i.e., $>0.4 \mathrm{mg} / \mathrm{kg} /$ day equivalent of prednisolone for $>15$ consecutive days).

Patients were followed up for 28 days. During the patients' ICU hospitalization, the following information was recorded: the results from clinical and routine laboratory examinations; pathological parameters, including blood tests, C-reactive protein (CRP) levels, blood pressure, heart rate, blood gases, respiratory rate, vasoconstrictor and/or inotropic drug needs, cardiac index and fluid needs; the pathogen antibiogram; the type and dose of antibiotics administered; and the disease severity indices. The disease severity indices were based on the acute physiology and chronic health evaluation (APACHE) II score and the sequential organ failure assessment (SOFA) score. Repeated blood cultures were collected every 2 days.
The aim of the present study was to investigate the potential association of baseline (upon sepsis diagnosis) levels of $\operatorname{IgM}, \operatorname{IgG}, \operatorname{IgA}$ and $\operatorname{IgE}$ and circulating WBCs and $\mathrm{T}$ and $\mathrm{B}$ cells subpopulations with the clinical and microbiological response of patients with sepsis or septic shock owing to bacteremia from CnS-GNB.

\section{Antibiotic susceptibility testing}

Gram-negative isolates were identified using VITEK 2 Advanced Expert System (bioMerieux, Marcy l'Etoile, France). MICs of meropenem, fosfomycin, tigecycline and ceftazidime/ avibactam were determined by Etest (bioMerieux), whereas, broth microdilution method was applied for colistin. Results were interpreted according to European Committee on Antimicrobial Susceptibility Testing (EUCAST) 2018 guidelines [19]. The presence of blaKPC, blaVIM, blaNDM and blaOXA-48 genes was confirmed by polymerase chain reaction (PCR).

\section{Definition of the outcomes}

Microbiological response was defined as two consecutive negative blood cultures for the isolated bacteria at 14 days after initiation of active antimicrobial therapy (i.e., test of cure visit). An early microbiological response was defined as negativity of blood cultures within 5 days of active antimicrobial treatment initiation. The initial clearance and recurrence of the pathogen within a 14-day period was classified as a treatment failure.

Clinical response was defined as the resolution of all clinical and laboratory signs of infection and sepsis at 14 days of active antimicrobial treatment for the isolated pathogen (i.e., test-of-cure visit). Resolution of infection signs included fever resolution, hemodynamic stabilization of patients with a reduction or discontinuation in vasoconstrictor and/or inotropic drugs, a normalization of the laboratory values of WBCs and coagulation parameters and significant reduction in CRP levels.

\section{Sample preparation and analysis}

We measured the levels of four Igs: $\operatorname{IgM}, \operatorname{IgG}, \operatorname{IgA}$ and $\operatorname{IgE}$. Circulating $\mathrm{T}$ and $\mathrm{B}$ cells levels were evaluated using fluorescence-activated cell sorting (FACS) and flow cytometry. Two $5-\mathrm{mL}$ blood samples were collected from each patient in a tube containing ethylenediamine tetraacetic acid (EDTA) as an anticoagulant at sepsis diagnosis. One sample was sent to the flow cytometry laboratory for analysis, while the other blood sample was sent to the microbiology laboratory for quantification of the Igs using the nephelometer method. Monoclonal antibody cell signalling, and erythrocyte lysis was used to prepare the blood sample for the lymphocyte count. The sample preparation time was approximately $45 \mathrm{~min}$. The sample analysis took approximately $20 \mathrm{~min}$ and was performed using the Navios EX Flow Cytometer (Beckman Coulter Life Science, Brea, CA, USA). The following lymphocyte subpopulations were measured during analysis: CD3 (T lymphocytes), 
CD4 (T helper cells), CD8 (cytotoxic T lymphocytes), CD3 ${ }^{+}$ $\mathrm{CD}^{2} 6^{+}$(NK cells $\mathrm{T}$ lymphocytes), $\mathrm{CD}^{+} \mathrm{CD}^{+} 6^{+}$(NK cells), $\mathrm{CD}^{+}{ }^{+} \mathrm{HLA}^{-D R}{ }^{+}, \mathrm{CD} 20$ (B lymphocytes) and $\mathrm{CD}^{+} \mathrm{CD} 20^{+}$. The ratio of CD4 to CD8 was also measured as a percentage of CD4 $\mathrm{T}$ cells divided by a percentage of CD8 T cells.

\section{Statistical methods}

Data normality was tested using the Kolmogorov-Smirnov and Shapiro-Wilk methods. For the continuous variables following a normal distribution, the independent samples $t$-test was used, while the Mann-Whitney U test was used for the variables not following a normal distribution. The McNemar test (crosstabs, binomial distribution, $\mathrm{Chi}^{2}$-tests) was used for the categorical variables while Kendall's tau-b test was used for the estimation of the bivariate microbiological vs. clinical response correlation). Covariance factors that possibly contributed to the final statistical result of the patients' microbiological and clinical response were studied using binary logistic regression analysis. Data were presented as the mean and standard deviation (SD) for the continuous variables following a normal distribution and as the median and interquartile range (IQR) for the continuous variables not following a normal distribution.

The following analyses were performed according to a predefined plan. First, statistical analyses were performed for the Ig values and the patients' microbiological and clinical responses, and then for the WBCs and the lymphocyte values and the patient's microbiological and clinical responses. The correlation of the microbiological and the clinical responses of the patients were then investigated. Finally, the binary logistic regression analysis examined the possible covariance contribution to the outcome in a backward stepwise method. Two logistic regression models were used with the microbiological and clinical responses as the outcome, or dependent variable. The statistical analyses of the data were performed using the SPSS version 23 for MAC (IBM SPSS Statistics for Macintosh, Armonk, NY, USA) and $\mathrm{P}<0.05$ was considered to be statistically significant.

\section{Results}

A total of 24 patients were included in the study: 16 males and eight females. Amongst them 10 had bacteremia from Klebsiella pneumonia, 12 from Acinetobacter baumannii and two from Pseudomonas aeruginosa. The microbiological and clinical responses of the patients are presented in Tables 1 and 2, respectively. There were 18 patients $(75 \%)$ with microbiological response and six patients $(25 \%)$ without microbiological response, while 16 patients presented clinical response (64\%) and eight patients $(36 \%)$ did not have clinical response. There were no statistically significant differences in age, sex, APACHE II and SOFA scores, CRP levels, and the presence of fever between patients with or without a microbiological or clinical response.

The results showed that there were no statistically significant differences in the levels of Igs between patients with or without a microbiological response: $\mathrm{P}=0.1$ for $\operatorname{IgA}, \mathrm{P}=0.7$ for $\operatorname{IgE}, \mathrm{P}=1$ for $\operatorname{IgM}$ and $\mathrm{P}=0.4$ for $\operatorname{IgG}$ (Table 1 , Fig. 1a). In addition, there were no statistically significant differences in the levels of Igs between patients with or without a clinical response: $\mathrm{P}=0.05$ for $\operatorname{IgA}, \mathrm{P}=0.6$ for $\operatorname{IgE}, \mathrm{P}=0.8$ for $\operatorname{IgM}$ and $\mathrm{P}=0.3$ for IgG (Table 2, Fig. 1b).

There were no statistically significant differences in the levels of WBCs, subpopulations of T and B lymphocytes and in the ratios of CD4 to CD8 between patients who exhibited or not a microbiological response: $\mathrm{P}>0.05$ for all variables (Table 1, Fig. 2a). There were also no statistically significant differences for all variables between patients with or without a clinical response: $\mathrm{P}>0.05$ (Table 2, Fig. 2b).

There was no statistically significant correlation between the microbiological responses and the clinical responses of patients $(\mathrm{P}=0.74$ for $\mathrm{McNemar}$ test and $\mathrm{P}=1(95 \%$ confidence interval: -0.2 - 0.2) for Kendall's tau-b test)). Specifically, 12 patients who presented with a microbiological response also showed a clinical response, while six patients who presented with a microbiological response did not have a clinical response. In total, 18 patients showed a microbiological response. Four patients who presented without a microbiological response showed a clinical response, while two patients did not present with a microbiological or a clinical response. Six patients showed an early microbiological response, although there was no statistically significant correlation between patients who showed an early microbiological response and patients who presented with a clinical response $(P=0.6$, Table 2$)$.

A subgroup analysis was also performed having divided patients into two age groups ( $\leq 60$ and $>60$ years old). In patients $\leq 60$ years old there was a statistically significant difference in CD-20 and CD5-CD20 B-lymphocytes values $(\mathrm{P}=0.048$ and $\mathrm{P}=0.047$, respectively) with higher values in patients without microbiological response ((median (IQR), number $/ \mathrm{mm}^{3}$ ), 148 (77 - 175) vs. 207 (183 - 340) for CD5-CD20 B-lymphocytes and 6 (2.7 - 17) vs. 23.5 (17 - 51) for CD-20 lymphocytes). In patients $>60$ years old IgA values were statistically significant different and higher in patients without microbiological response ( $\mathrm{P}=0.037$, (median (IQR), mg/dL), 329 (317 - 330) vs. $184(106-227))$. There were no statistically significant differences in Igs and lymphocytes subpopulations in the clinical response of patients between the two age groups.

The binary logistic regression analysis revealed no factors associated with the microbiological or clinical responses of patients at $\mathrm{P}<0.1$ and, thus, a multivariate analysis was not performed. The variables that were examined in our models, which included age, sex, APACHE II and SOFA scores, levels of WBCs, CRP levels, levels of Igs, levels of lymphocyte subpopulations and the ratio of CD4 to CD8, were based on scientific knowledge and considered potential collinearity. For both the microbiological response and the clinical response, an adjusted analysis for patients with a SOFA score $\leq 8$ and a SOFA score $>8$ was performed; the analysis revealed no differences at $\mathrm{P}<0.1$. Only one patient died within the 28-day follow-up.

\section{Discussion}

In the present study, the levels of $\operatorname{IgA}, \operatorname{IgM}, \operatorname{IgG}$ and $\operatorname{IgE}$ were 
Table 1. Cohort Patients' Characteristics in Relation to Microbiological Response

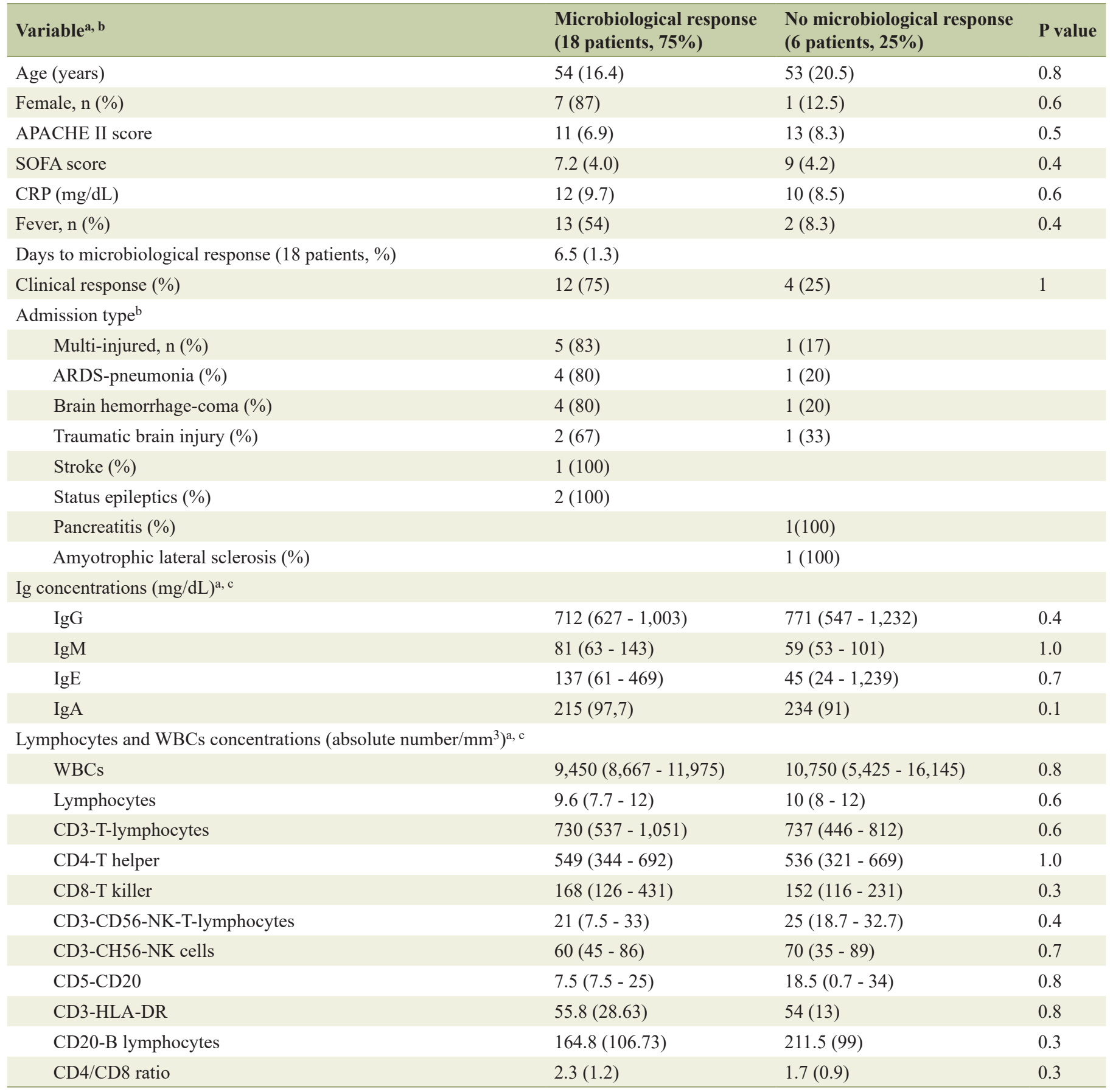

${ }^{a}$ Continuous data presented as mean and SD when normally distributed. ${ }^{b}$ Categorical data presented as number (proportion). ${ }^{c}$ Continuous data presented as median and interquartile range when not normally distributed. APACHE II: Acute Physiology and Chronic Health Evaluation II; SOFA: Sequential Organ Failure Assessment; CRP: C-reactive protein; Ig: immunoglobulin; WBCs: white blood cells; NK: natural killer; HLA: human leukocyte antigen; SD: standard deviation.

measured in patients with documented Gram-negative bacteremia and sepsis or septic shock from CnS-GNB. The analysis showed there were no statistically significant differences in the levels of Igs between patients with or without a microbiological response or clinical response. Furthermore, there were no statistically significant differences in the levels of circulating subpopulations of $\mathrm{T}$ and $\mathrm{B}$ cells between patients with or without a microbiological or clinical response.

To our knowledge, this was the first study to evaluate the relationship between the levels of Igs, WBCs and lymphocytes 
Table 2. Cohort Patients' Characteristics in Relation to Clinical Response

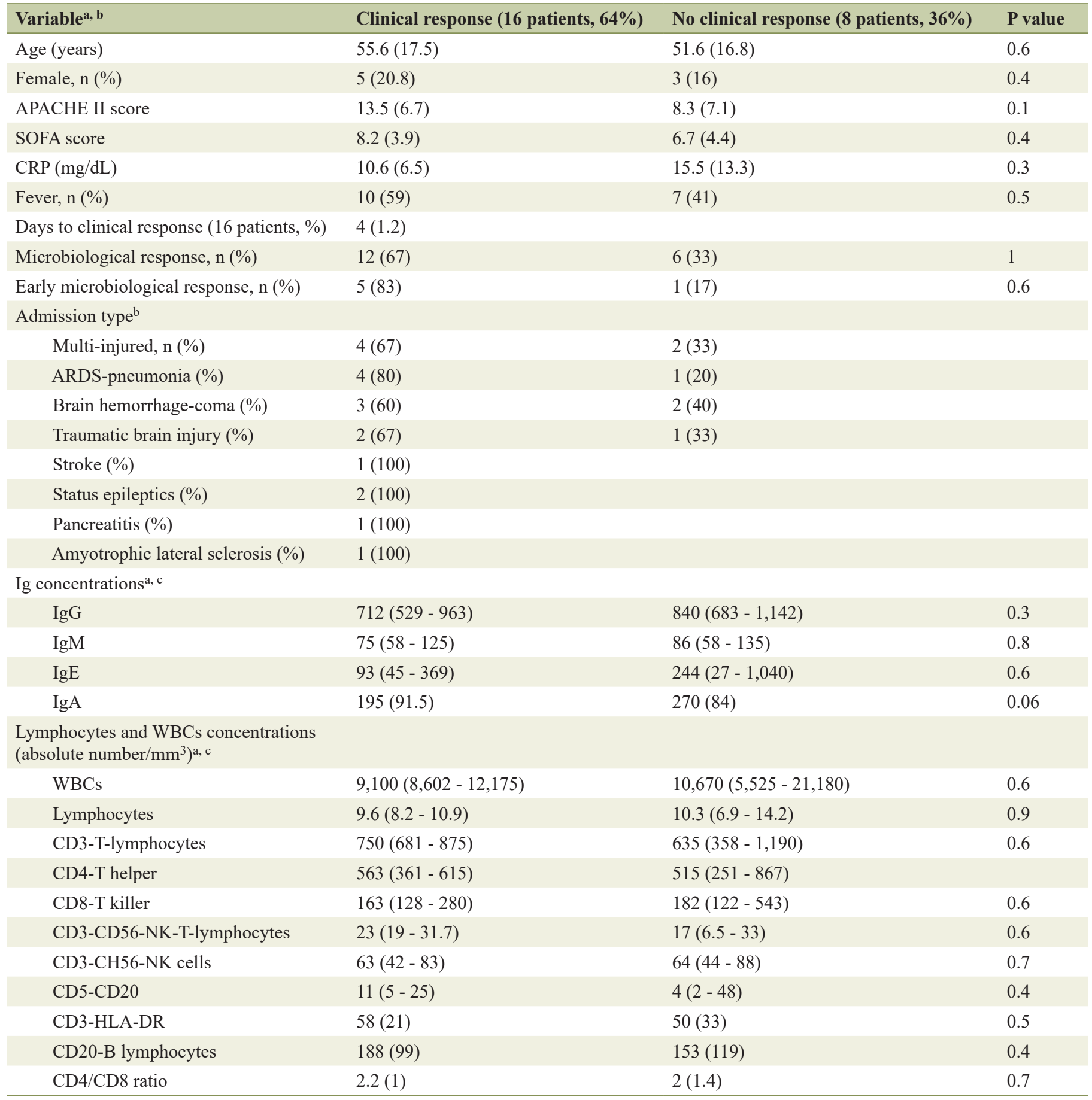

aContinuous data presented as mean and SD when normally distributed. ${ }^{b}$ Categorical data presented as number (proportion). ${ }^{\mathrm{c}} \mathrm{Continuous} \mathrm{data}$ presented as median and interquartile range when not normally distributed. APACHE II: Acute Physiology and Chronic Health Evaluation II; SOFA: Sequential Organ Failure Assessment; CRP: C-reactive protein; Ig: immunoglobulin; WBCs: white blood cells; NK: natural killer; HLA: human leukocyte antigen; SD: standard deviation.

and the microbiological and clinical responses in patients with sepsis or septic shock from CnS-GNB bacteremia. Low concentrations of Igs are seen in most adult patients with sepsis; the most common quantitative Ig abnormality that is seen in sepsis involves low levels of $\mathrm{IgG}$, while according to the literature, the nadir Igs levels are often seen within 3 days of a diagnosis of sepsis [8, 14].

Although, low levels of $\operatorname{IgG}$ alone in patients with sepsis 
$\mathbf{a}$

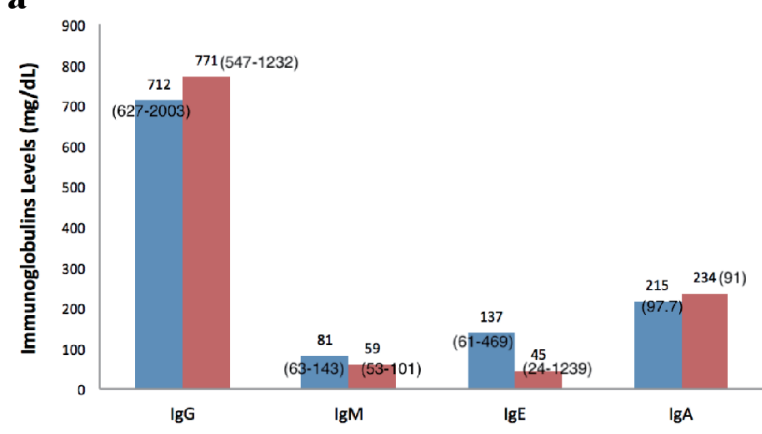

b

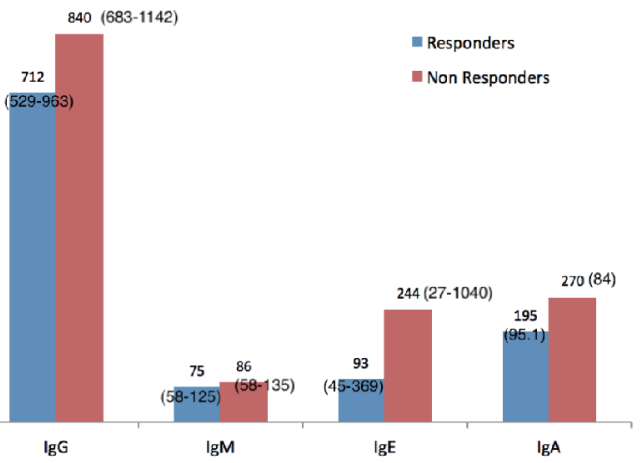

Figure 1. Immunoglobulins levels and patients' microbiological (a) and clinical response (b). Data are presented as mean and SD when normally distributed and as median and interquartile range when not normally distributed. SD: standard deviation.

do not increase the risk of death, low levels of multiple endogenous Igs have been found to increase the mortality risk in these patients $[8,12-14]$.
In our study, we found that the levels of the four Igs that were evaluated were in the normal range. A recent retrospective observational study by Loeches et al suggested that there

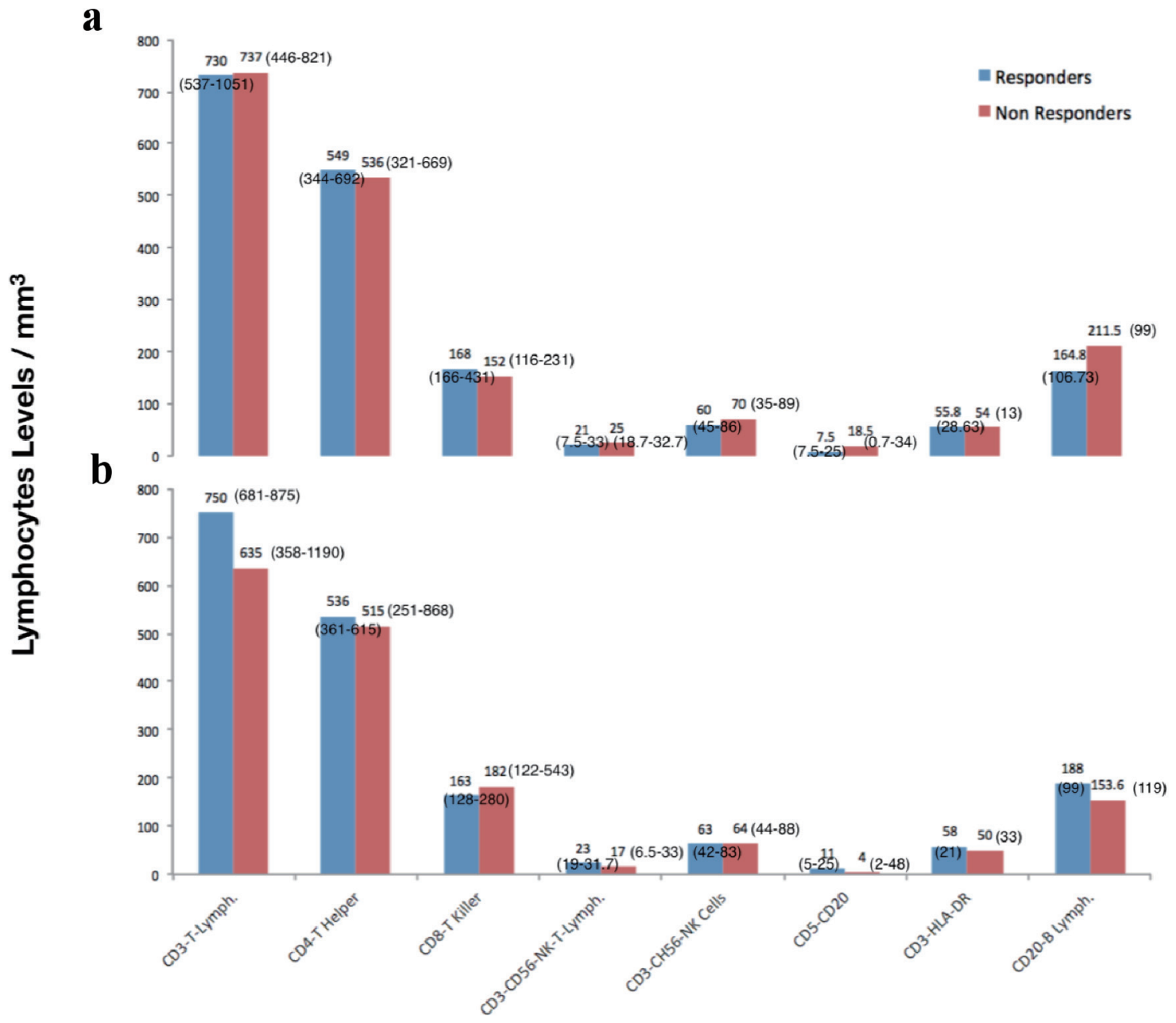

Figure 2. Lymphocytes subpopulations levels and patients' microbiological (a) and clinical response (b). Data are presented as mean and SD when normally distributed and as median and interquartile range when not normally distributed. SD: standard deviation. 
was an association between low levels of Igs and mortality risk in patients with sepsis who had less severe organ dysfunction and SOFA scores $<8$ [20]. Our study did not show this correlation considering patients' microbiological and clinical response. However, this may be due to the small number of patients involved, as well as the observational design of the study. In fact, we found that the SOFA scores were similar and there were no statistically significant differences between patients with or without microbiological and clinical responses. Ultimately, the primary outcome of our study was not mortality risk, and therefore our results were not exactly comparable with the results of the study by Loeches et al [20].

Early T-cell lymphopenia that is observed in patients with sepsis is due to cell migration from the systemic circulation to the site of infection [21]. Apoptosis of $\mathrm{T}$ and $\mathrm{B}$ lymphocytes has been demonstrated in many models of sepsis, post-mortem analysis of septic patients and in the circulation of patients with septic shock while persistent lymphopenia is a risk factor for ICU-acquired infections and death [22, 23].

Numerous experimental studies of sepsis in mice have shown that $\mathrm{CD}^{+} \mathrm{T}$ lymphocytes play a key role in reducing infection and abscess formation [7]. Cellular subsets, especially different $\mathrm{CD}^{+} \mathrm{T}$ lymphocytes subsets, play a critical role in response to microbial challenge and may contribute to nosocomial infection susceptibility $[22,24]$. $\mathrm{CD} 8^{+} \mathrm{T}$ lymphocytes are effector cytotoxic cells, which were found to be decreased in patients with septic shock at ICU admission, while NK cells which are also affected by lymphopenia, are potent producers of proinflammatory mediators and also present effector cytotoxic activity [24]. B lymphocytes can differentiate into Ig-secreting plasma cells. They have an inverse correlation between count and survival, but this relation affects heterogeneously their subpopulations [24].

In our study, the mean/median values of T and B cells subpopulations, especially CD4 T-helper cells, CD8-killer cells (cytotoxic T lymphocytes), NK cells and CD3 (T lymphocytes), were not statistically significant between patients with or without a microbiological or clinical response. However, we found that the mean/median values of the CD8 (T-killer), CD4 (T helper), NK cells and CD3 (T lymphocytes) were lower than normal. These results were expected and were consistent with previous studies that found that, during sepsis, the lymphocytes decrease the expression of genes associated with the inflammatory response and increase the expression of genes associated with apoptosis, while sepsis-induced immunosuppression is significantly due to the depletion of the memory $\mathrm{B}$ cell compartment $[7,12]$.

The mean CRP values did not show statistically significant differences between patients with or without microbiological or clinical responses. We would expect WBCs and CRP levels to be lower in patients who had a microbiological and/or clinical response compared with patients without response. However, the small sample size may have contributed to this result. In addition, the sampling time may have played a role because the immune system needs time to adapt to changes, although this would be quite unlikely because the blood samples were drown upon sepsis or septic shock diagnosis.

In our study, six patients with a microbiological response did not show a clinical response, while four patients without a microbiological response showed a clinical response. There are various possible causes for these findings. For example, a patient could have had a new infection from a different pathogen that had not yet been identified, and thus, the patient clinically deteriorated despite the microbiological response to the original bacteria. In addition, the clinical improvement of a patient could have occurred at a slower rate than the patient's microbiological response. The clinical improvement of a patient could also have been related to the termination of the systemic inflammatory response caused by factors other than infection (severity of the underlying disease), although the bacteremia had not been eradicated.

When patients were divided into two age groups $(\leq 60$ and $>60$ years old) there were statistically significant differences in some lymphocytes subpopulations and in IgA values, but as only 24 patients were included in our study the results are questionable.

Our study had many limitations. The most important limitation was the small size of our sample ( 24 patients). Although this was a prospective study, it was observational, and therefore, the possibility of bias in the selection of patients and the assessment of the various parameters and objectives of the study cannot be ruled out. In addition, the patients had similar, but different susceptibilities to the various classes of antibiotics and antibiotic treatments used. Although all patients had a CnSGNB infection, the study included patients with different bacterial infections, which may have affected the severity of the disease and the susceptibilities to the antibiotics. Finally, as only 24 patients were included in the study the results of the binary logistic regression analysis may be questionable.

In conclusion, in patients with sepsis or septic shock from CnSGNB, there were no differences in the levels of Igs, circulating WBCs and T and B cells subpopulations between those with microbiological or clinical response and non-responders. The immune system of patients with sepsis is affected by a variety of factors, and thus, further studies, laboratory and clinical models are needed. Future studies will help develop targeted therapies to treat immunosuppression or provide immunostimulation.

\section{Acknowledgments}

The investigators acknowledge the microbiology, hematology and intensive care departments and staff that participated in the research project (General University Hospital of Patras).

\section{Financial Disclosure}

This study was supported by General University Hospital of Patras fundings.

\section{Conflict of Interest}

The authors have no conflict of interest to disclose. 


\section{Informed Consent}

The data were collected after consent was obtained from the closest relative, as the patients were unable to provide consent due to the severity of their disease.

\section{Author Contributions}

DA oversaw data collection, reviewed the literature, analyzed and interpreted the data, and wrote the manuscript. KL engaged in data collection, data interpretation, and contributed to drafts of the manuscript. FF contributed to data collection and literature review. KA contributed to the literature review and contributed to drafts of the manuscript. AS contributed to data collection and reviewed the manuscript. AN engaged in data collection. SFA designed the study, oversaw data collection, reviewed the literature and provided critical reviews of the manuscript.

\section{Data Availability}

The data supporting the findings of this study are available from the corresponding author upon reasonable request.

\section{References}

1. Fleischmann C, Scherag A, Adhikari NK, Hartog CS, Tsaganos T, Schlattmann P, Angus DC, et al. Assessment of global incidence and mortality of hospital-treated sepsis. Current Estimates and Limitations. Am J Respir Crit Care Med. 2016;193(3):259-272.

2. Reinhart K, Daniels R, Kissoon N, Machado FR, Schachter RD. Recognizing sepsis as a global health priority - A WHO Resolution. N Engl J Med. 2017;377:414417.

3. Vincent JL, Rello J, Marshall J, Silva E, Anzueto A, Martin CD, Moreno R, et al. International study of the prevalence and outcomes of infection in intensive care units. JAMA. 2009;302(21):2323-2329.

4. Leentjens J, Kox M, van der Hoeven JG, Netea MG, Pickkers P. Immunotherapy for the adjunctive treatment of sepsis: from immunosuppression to immunostimulation. Time for a paradigm change? Am J Respir Crit Care Med. 2013;187(12):1287-1293.

5. Seymour CW, Liu VX, Iwashyna TJ, Brunkhorst FM, Rea TD, Scherag A, Rubenfeld G, et al. Assessment of clinical criteria for sepsis: for the third international consensus definitions for sepsis and septic shock (Sepsis-3). JAMA. 2016;315(8):762-774.

6. Cohen J, Vincent JL, Adhikari NK, Machado FR, Angus DC, Calandra T, Jaton K, et al. Sepsis: a roadmap for future research. Lancet Infect Dis. 2015;15(5):581-614.

7. Rimmele T, Payen D, Cantaluppi V, Marshall J, Gomez H, Gomez A, Murray P, et al. Immune cell phenotype and function in sepsis. Shock. 2016;45(3):282-291.
8. Shankar-Hari M, Singer M, Spencer J. Can concurrent abnormalities in free light chains and immunoglobulin concentrations identify a target population for immunoglobulin trials in sepsis? Crit Care Med. 2017;45(11):1829-1836.

9. Almansa R, Wain J, Tamayo E, Andaluz-Ojeda D, Martin-Loeches I, Ramirez P, Bermejo-Martin JF. Immunological monitoring to prevent and treat sepsis. Crit Care. 2013;17(1):109.

10. Peters van Ton AM, Kox M, Abdo WF, Pickkers P. Precision immunotherapy for sepsis. Front Immunol. 2018;9:1926.

11. Delano MJ, Ward PA. Sepsis-induced immune dysfunction: can immune therapies reduce mortality? J Clin Invest. 2016;126(1):23-31.

12. Shankar-Hari M, Madsen MB, Turgeon AF. Immunoglobulins and sepsis. Intensive Care Med. 2018;44(11):19231925.

13. Bermejo-Martin JF, Rodriguez-Fernandez A, HerranMonge R, Andaluz-Ojeda D, Muriel-Bombin A, Merino P, Garcia-Garcia MM, et al. Immunoglobulins IgG1, IgM and IgA: a synergistic team influencing survival in sepsis. J Intern Med. 2014;276(4):404-412.

14. Shankar-Hari M, Culshaw N, Post B, Tamayo E, AndaluzOjeda D, Bermejo-Martin JF, Dietz S, et al. Endogenous IgG hypogammaglobulinaemia in critically ill adults with sepsis: systematic review and meta-analysis. Intensive Care Med. 2015;41(8):1393-1401.

15. Giamarellos-Bourboulis EJ, Tziolos N, Routsi C, Katsenos C, Tsangaris I, Pneumatikos I, Vlachogiannis G, et al. Improving outcomes of severe infections by multidrugresistant pathogens with polyclonal IgM-enriched immunoglobulins. Clin Microbiol Infect. 2016;22(6):499-506.

16. Shankar-Hari M, Fear D, Lavender P, Mare T, Beale R, Swanson C, Singer M, et al. Activation-associated accelerated apoptosis of memory B cells in critically ill patients with sepsis. Crit Care Med. 2017;45(5):875-882.

17. Shankar-Hari M, Datta D, Wilson J, Assi V, Stephen J, Weir CJ, Rennie J, et al. Early PREdiction of sepsis using leukocyte surface biomarkers: the ExPRES-sepsis cohort study. Intensive Care Med. 2018;44(11):18361848.

18. Drewry AM, Samra N, Skrupky LP, Fuller BM, Compton SM, Hotchkiss RS. Persistent lymphopenia after diagnosis of sepsis predicts mortality. Shock. 2014;42(5):383391.

19. The European Committee on Antimicrobial Susceptibility Testing (EUCAST). Breakpoint tables for interpretation of MICs and zone diameters. Version 9.0, 2019. Available at: http://www.eucast.org. [accessed 15 June 2019].

20. Martin-Loeches I, Muriel-Bombin A, Ferrer R, Artigas A, Sole-Violan J, Lorente L, Andaluz-Ojeda D, et al. The protective association of endogenous immunoglobulins against sepsis mortality is restricted to patients with moderate organ failure. Ann Intensive Care. 2017;7(1):44.

21. Krabbe KS, Bruunsgaard H, Qvist J, Fonsmark L, Moller $\mathrm{K}$, Hansen CM, Skinhoj P, et al. Activated T lymphocytes disappear from circulation during endotoxemia in humans. Clin Diagn Lab Immunol. 2002;9(3):731-735.

22. Denstaedt SJ, Singer BH, Standiford TJ. Sepsis and no- 
socomial infection: patient characteristics, mechanisms, and modulation. Front Immunol. 2018;9:2446.

23. Adrie C, Lugosi M, Sonneville R, Souweine B, Ruckly S, Cartier JC, Garrouste-Orgeas M, et al. Persistent lymphopenia is a risk factor for ICU-acquired infections and for death in ICU patients with sustained hypotension at admission. Ann Intensive Care. 2017;7(1):30.

24. de Pablo R, Monserrat J, Prieto A, Alvarez-Mon M. Role of circulating lymphocytes in patients with sepsis. Biomed Res Int. 2014;2014:671087. 